\title{
Chinese Adolescents' Conflict with Parents and Its Relationship with Their Psychological Well-Being
}

\author{
Jianjin Liu \\ Guangdong University of Foreign Studies, Guangzhou, China \\ Email: liujianjinljj@126.com
}

How to cite this paper: Liu, J.J. (2020) Chinese Adolescents' Conflict with Parents and Its Relationship with Their Psychological Well-Being. Open Access Library Journal, 7: e6256.

https://doi.org/10.4236/oalib.1106256

Received: May 23, 2020

Accepted: August 2, 2020

Published: August 5, 2020

Copyright () 2020 by author(s) and Open Access Library Inc.

This work is licensed under the Creative Commons Attribution International License (CC BY 4.0).

http://creativecommons.org/licenses/by/4.0/

\section{(c) (i) Open Access}

\begin{abstract}
This paper investigated 125 Chinese adolescents' daily conflicts with their parents through blogs within 2 weeks on a platform specifically designed for this study. BSI (Brief Symptom Inventory) scores were also collected to measure their psychological well-being. 3-way ANOVAs (age $\times$ gender $\times$ BSI group) were conducted on variables of parent-child conflicts (frequency, intensity, resolution, fairness and feelings towards resolution, justification). The main findings are: 1) adolescents from the low-BSI group felt less unfair and upset or depressed to the resolution of the conflicts, compared to those from the high-BSI group. In other words, when adolescents felt more fair and happy to the resolution to the conflicts, they have a healthier status of psychological well-being; 2) frequency and intensity of conflicts in general had no significant correlation with adolescents' psychological well-being, but there is an interaction between gender and BSI score on intensity; 3 ) there is a significant difference between low- and high-BSI groups in conflicts concerning personal issues or choices, which indicates low-BSI group (with a higher level of psychological well-being) negotiated more on personal issues with their parents; 4) there is an interaction between adolescents' age and BSI in personal justification and an interaction between gender and BSI in psychological justification. Implications and suggestions on education related to adolescents' psychological well-beings are discussed.
\end{abstract}

\section{Subject Areas}

Developmental Psychology, Parent-Child Conflict

\section{Keywords}

Chinese Adolescents, Parent-Child Conflicts, Justification, Psychological Well-Being 


\section{Introduction}

G. Stanley Hall, a famous American psychologist and educationist, compared adolescence to "stormy period". He believed that the biological change led to dramatic change of adolescents' psychology, and that parent-child conflict was the only way for adolescents to become independent. But later scholars found that parent-child conflict may have a negative impact on the mental health of adolescents. For example, it was found that parent-child conflict was associated with low life satisfaction, self-esteem, hopelessness and mental illness (Shek, 1998) [1]. Ge et al. (1994) also found that the increased frequency of parent-child conflict can lead to high levels of depression and anxiety in adolescents, especially girls [2]. Meanwhile, adolescents who experienced frequent and tense parent-child conflicts will have a lower sense of intimacy with their parents and often form a negative perception of family functions (Daniel \& Shek, 1999) [3]. Grotevant and Cooper (1986) found that adolescents who get parents' support in parent-child relationship can better seek self-identity, while adolescents who have conflict with their parents are more likely to have various emotional and behavioral problems [4].

Chinese scholars have made extensive research on the content, intensity, development and change of parent-child conflict, the factors that influencing parent-child conflict and its possible influence on the social and emotional development of adolescents. For instance, Fang et al. (2003) found that parent-child conflict has a relatively consistent correlation with adolescents' depression and problematic behaviors, that is, adolescents who conflict with their parents show the most problematic behaviors and depression, followed by adolescents who conflict with their single parents, and adolescents who have no conflict with their parents show the least problematic behaviors and depression [5]. Yang and $\mathrm{Wu}$ (2014) found that there was a significant positive correlation between depression/anxiety and parent-child conflict, which was closely related to all dimensions of parenting style. This shows that the more reasonable parenting style is, the fewer conflicts with children occur, and the less likely children are to have depression and anxiety [6]. Yin and Shi (2010) believed that generally speaking, low-level parent-child conflict was conducive to the development of youth identity and sociality, but the research also found that there is a significant correlation between parent-child conflict and adolescents' problematic behavior, crime, drug use, sexual behavior, poor academic performance, etc. [7].

In summary, though most scholars agree that parent-child relationship has an influence on adolescents' psychological development, they split on the characteristics of the influence (positive or negative?) and what aspect of parent-child relationship will influence adolescents (frequency, intensity, content, or solution to the conflicts?). Meanwhile, most studies employed questionnaire as the main method, and questionnaire has its own limitations. For example, how adolescents defend for themselves when conflict with their parents is seldom studied because of the limitation of questionnaire. Based on these analyses, this research 
used online diary to collect first-hand data to further explore how parent-child conflicts may influence adolescents' psychological well-being. More specifically, 3-way ANOVAs (age $\times$ gender $\times$ BSI group) were conducted to test the following hypotheses:

$\mathrm{H1}$ : the frequency and intensity will have a negative influence on adolescents' psychological well-being;

$\mathrm{H} 2$ : how adolescents feel about the resolution to the conflicts will greatly influence adolescents' psychological well-being;

H3: parent-child conflict in different domains may have different influences on adolescents' psychological well-being;

H4: justifications adolescents employed to defend themselves may have significant correlation with adolescents' psychological well-being;

H5: gender and age matter in all assumptions.

\section{Method}

\subsection{Location and Participants}

125 adolescents from public middle schools (23 males and 33 females, $M$ age = 13.9) and public high schools ( 32 males and 37 females, $M$ age $=16.9$ ) were recruited in Guangzhou, the capital city of Guangdong Province in China.

\subsection{Procedure}

The author visited several middle schools and high schools to invite students to take part in the research. All participants got assent from their parents and were promised a gift when they finished online blogs and questionnaires.

The recruited adolescents were asked to register in a blog site specifically designed for this study (http://tell.dezend.cn), and write blogs there for two weeks. During these two weeks, they were required to write down any conflicts (minor or major disagreement) happening between their parents and them. For each conflict, the adolescents were asked to 1) rate the intensity of the conflict, 2) explain why they think they are right when they disagree with their parents, 3) tell how the conflict was resolved, 4) rate the fairness level of the conflict resolution, and 5) evaluate the feelings towards the conflict resolution. In addition, these adolescents were also required to finish the questionnaire BSI of (Brief Symptom Inventory).

During these two weeks, participants received automatic reminding message of writing blogs from the platform every other day. The research assistant checked whether the adolescents recorded conflicts regularly from time to time and would email to or call them if they didn't visit the platform.

\subsection{Measures}

All measures used in this research were taken from prior work conducted in Japan (Smetana, Araki, \& Nakaue, 2014) [8] and China (Chen-Gaddini, 2012 [9]; Helwig, Yang, Nucci, Yun, \& To, 2009 [10]). 
Adolescents daily conflicts with parents. A novel feature of the present study was that we used an online platform to have adolescents record real-time daily conflicts with their parents in 2 weeks. The measures for the conflicts can be found in previous research (Chen-Gaddini, 2012 [9], Yau \& Smetana, 1996 [11], 2003 [12]) that used the interview to make adolescents recall their conflicts with parents in the past two weeks (conflicts were defined as any minor or major disagreement). To evaluate the intensity of each conflict, adolescents were required to rate each conflict on a scale ranging from 1 (mild) to 5 (very severe). The total number of all conflicts each participant generated were summed to obtain a measure of frequency of conflicts. Considering the different total number of conflicts generated by participants, ratings of conflict intensity were averaged to obtain a mean intensity for each participant.

To assess participants' justifications for the act's wrongness, for each conflict, participants were asked: "Why do you think it is right for you to do XXX?" Answers to this question were coded into 17 categories $^{1}$ and then collapsed into five superordinate categories: 1) moral justification, involving concerns for others' welfare, feelings of duty, and appeals to fairness; 2) conventional justification, involving appeals to authority and custom, avoidance of punishment, references to politeness or courtesy, and concerns for responsibility; 3) psychological justification, about friendship, interpersonal relationships, and adolescents' psychological development; 4) personal justification, including references to autonomy seeking and the act's permissibility, and the recognition that the act has minimal or inconsequential effects on self or others; and 5) pragmatic justification, including concerns for personal comfort or health, and references to practical needs and consequences. Justifications that could not be coded in these categories were coded as other. Interrater reliability of superordinate justification categories was kappa $=0.80$. To handle the different numbers of justifications given, responses were coded as proportions of adolescents' total justification responses.

Finally, participants were asked how each conflict was resolved, and their answers about conflict resolutions were coded into one of four categories: 1) adolescent won the conflict, 2) parents won the conflict, 3) joint resolution, or 4) no resolution. To assess the fairness of each resolution, they were asked to rate on a scale ranging from 1 (not fair at all) to 5 (very fair). Participants were also asked to rate their feelings toward each resolution on a scale ranging from 1 (very negative) to 5 (very positive).

Brief Symptom Inventory (BSI). The adolescents' psychological well-being in the study was assessed using the BSI (Derogatis, 1994 [13]; Derogatis \& Spencer, 1982 [14]) in a commercially available Chinese version. BSI includes 53 items concerning somatization (11 items), obsessive-compulsive disorder (6 items), interpersonal sensitivity ( 4 items), depression ( 6 items), anxiety (6 items), hostility (5 items), phobic anxiety (5 items), paranoia (5 items) and psychoticism (5 items).

${ }^{1}$ The coding of the adolescents' justification for conflicts was based on categories used in previous studies on adolescent-parent conflicts about everyday activities (Chen-Gaddini, 2012; Yau \& Smetana, 1996; Yau \& Smetana, 2009). 
Participants were asked to indicate how much they were distressed by each problem in the past 7 days using a scale ranging from 0 (not at all) to 4 (extremely). The scales of hostility, obsessive-compulsiveness, psychoticism, paranoid ideation, and phobia anxiety provide indications of severe psychological disorders that were not the focus of the present study and were not included in any analyses. In this study, only four dimensions of data were used: somatization, anxiety, depression and interpersonal sensitivity. Internal consistency (Cronbach's alpha) of the four scales in the present study were 0.83 (Depression), 0.80 (Anxiety), 0.72 (Interpersonal sensitivity), and 0.76 (somatization), respectively; and with an overall average alpha across scales of 0.78 .

\section{Analysis and Results}

The frequency, intensity of parent-child conflicts, justification and resolution to the conflicts and fairness and feelings towards resolution were analyzed between the two age groups by employing 2 (Age: early adolescents vs. late adolescents) $\times$ 2 (gender) $\times 2$ (BSI: high, low) ANOVAs. The main results are as followings:

1) Frequency and intensity of conflicts in general had no significant correlation with adolescents' psychological well-being, but there is an interaction between gender and BSI on the intensity of conflicts, $\mathrm{F}(1,82)=6.87, \mathrm{p}<0.05$, as shown in Figure 1. This indicates that intensity of conflicts had different influences on BSI on male and female adolescents. For male adolescents, higher intensity correlated with higher BSI score, i.e., when male adolescents had a more intensive conflict with their parents, they had a lower level of psychological well-being; there was no significant difference between high- and low BSI groups on intensity of conflicts in female adolescents.

2) There was significant BSI main effect on the fairness and feelings to conflict resolution, $\mathrm{MD}=-0.34,-0.37, \mathrm{~F}(1,82)=8.67, \mathrm{p}<0.01,0.05$. This shows that adolescents in the low BSI group (i.e. those with higher level of psychological well-being) felt more fair and have more positive views on the solutions than those in the high BSI group.

3) There was a gender main effect on personal justification for conflicts, $F(1$, $83)=5.38, \mathrm{p}<0.05$. This shows that male adolescents used significantly more personal justifications (43\%) than female adolescents $(32 \%)$ when they tried to defend for themselves in parent-child conflicts. There also existed an interaction between age and BSI groups, $\mathrm{F}(1,83)=8.17, \mathrm{p}<0.01$, which is shown in Figure 2 . The lower BSI group of early adolescents used more personal justification, while the higher BSI group used fewer personal justifications. On the contrary, the lower BSI group of late adolescents used fewer personal justifications, while the higher BSI group used more personal justifications. This is very interesting result, which indicates that more personal justifications were correlated to better psychological well-being, fewer ones were correlated to worse psychological well-being in early adolescents; on the contrary, when late adolescents used more personal justifications, they had worse psychological well-being, fewer personal justifications with better psychological well-being. 
4) There was an interaction between gender and BSI scores on psychological justification, $\mathrm{F}(1,82)=5.77, \mathrm{p}<0.05$, as shown in Figure 3 . This shows that the use of psychological justification had significantly different influences on their psychological well-being in male and female adolescents: for male adolescents, a high proportion of psychological justification was correlated to high BSI (i.e. a low level of psychological well-being), while there was no significant difference in their psychological well-being no matter female adolescents used more or fewer psychological justifications. This is quite interesting again. Psychological justification mainly includes the influence of behavior on interpersonal relationship (such as friendship and family affection), the developmental characteristics of teenagers' psychology, behavior and personality.

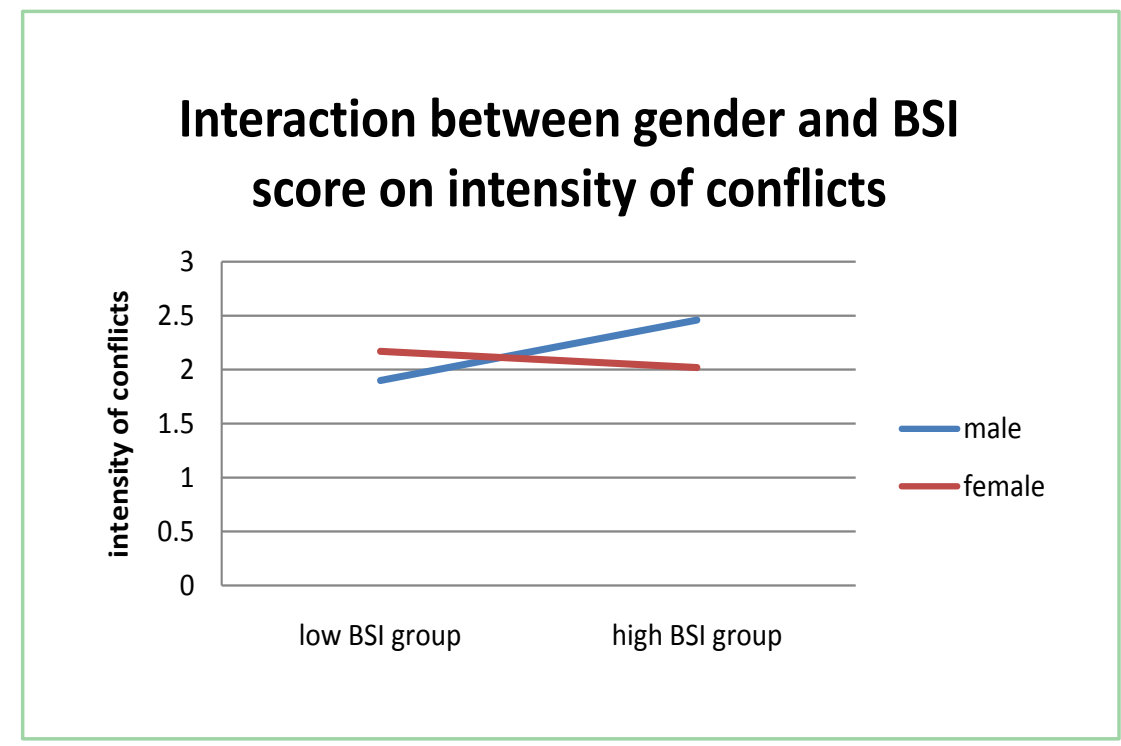

Figure 1. Interaction between gender and BSI score on intensity of conflicts.

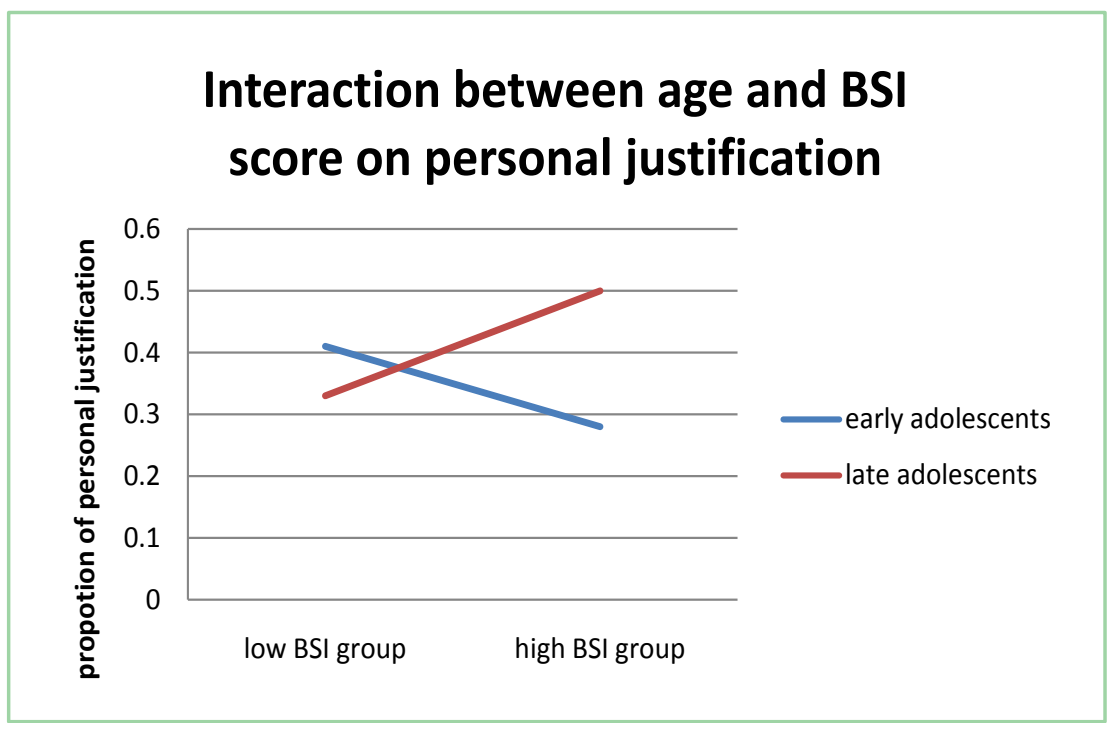

Figure 2. Interaction between age and BSI score on personal justification. 


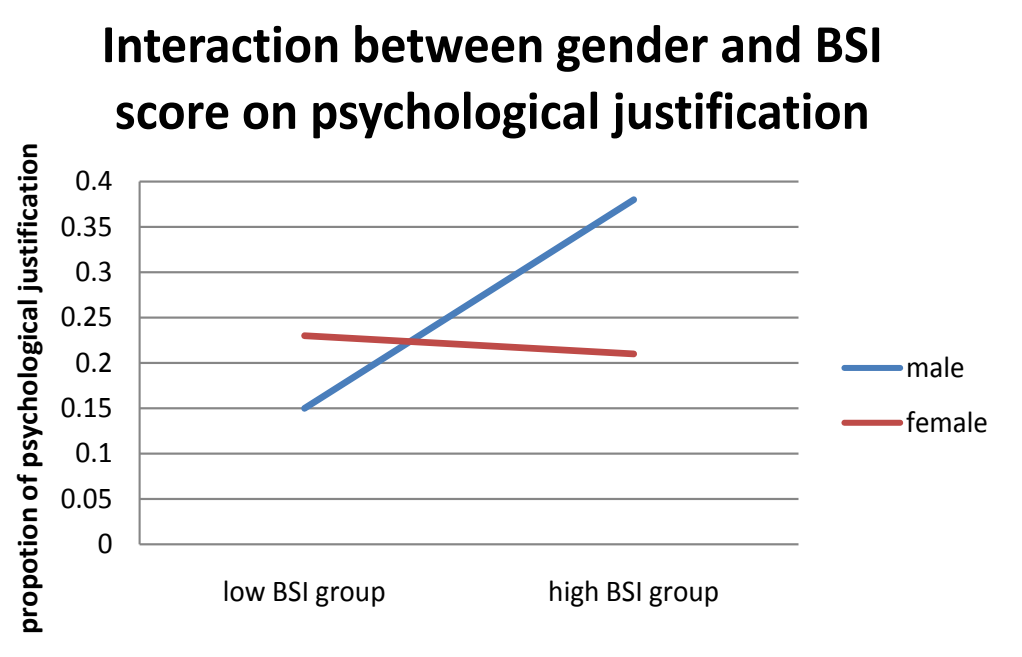

Figure 3. Interacton between gender and BSI score on psychological justification.

5) There is a significant difference between low-BSI group and high-BSI group in conflicts concerning personal issues or choices, $\mathrm{MD}=0.16, \mathrm{~F}(1,82)=9.30, \mathrm{p}$ $<0.01$. This means low-BSI group (with a higher level of psychological well-being) negotiated more on personal issues with their parents. It proves that parent-child conflicts are not necessarily bad for adolescents' psychological development; on the contrary, some conflicts may be beneficial to adolescents (just like conflicts over personal issues).

\section{Discussion and Implication}

\subsection{Parent-Child Conflicts}

Chinese culture advocates harmony and believes that conflict will destroy the relationship between people. Therefore, people always try to avoid conflicts in order to maintain a good interpersonal relationship. This concept is also reflected in the understanding of parent-child conflict: it's widely believed in China that the conflict between children and parents will inevitably lead to the deterioration of parent-child relationship, and also affect the healthy development of children and adolescents. Therefore, parents always view conflicts in a negative way, and try their best to avoid parent-child conflict. However, our results show that parent-child conflict does not necessarily lead to the deterioration of parent-child relationship or affect the psychological development of adolescents. On the contrary, some conflicts may be beneficial to teenagers' mental health. For example, the conflicts on clothing, food and other personal events are beneficial to teenagers' psychological well-being (with a lower BSI score).

To a certain extent, parent-child conflict expresses the requirements of adolescents' autonomous development, which is an important part of adolescents' psychological well-being. Sternberg and Silverberg (1986) believed that autonomy included de-idealization, regarding parents as ordinary people, independence from parents and individualization [15]. After entering puberty, the need 
for independence increases, and family relationships are transformed from more hierarchical relationships to more egalitarian relationships. In our research, early adolescents and late adolescents had significant difference in resolution to conflicts. More specifically, late adolescents won more in conflicts than early adolescents did, $\mathrm{MD}=0.20, \mathrm{~F}(1,82)=12.35, \mathrm{p}<0.01$; while early adolescents used more joint resolutions than late adolescents did, $\mathrm{MD}=0.34, \mathrm{~F}(1,82)=5.73, \mathrm{p}<$ 0.01 . Children's increasing demands on autonomy make them no longer absolutely obey their parents, but tend to express their own views and feelings. Wang (2006) found that in some daily life events, such as the choice of hair style, clothing and leisure style, teenagers' requirements for independence were increasing, and their dissatisfaction with their parents' authority was also increasing [16]. Meanwhile, they became more willing to oppose their parents' opinions directly and took a more acceptable attitude towards the differences with their parents. Smetana (1989) believed that adolescents' appeal for autonomy in individual events reflected adolescents' development of autonomy [17]. This is also one of the manifestations of teenagers' mental health development.

Lin (2002) believed that the pursuit of independence was an important task in the process of adolescent socialization [18]. In other words, without the development of teenagers' autonomy and independence, their socialization tasks cannot be completed, or cannot be fully completed. This will greatly affect the psychological development of adolescents and their future life. Therefore, as a promoter of the socialization of children and adolescents, parents are suggested temporarily put aside their "face" and authority, and give children and adolescents more autonomy in personal events and other fields. On the one hand, it can reduce parent-child conflict, and more importantly, it can promote the development of adolescents' autonomy and independence, thus promoting their psychological well-being.

\subsection{Resolution to Parent-Child Conflicts}

Our study found that the number and intensity of conflict had no significant impact on the general psychological well-being adolescents. This is different from our general understanding of parent-child conflict and psychological well-being. We generally believe that the more frequent parent-child conflict, the greater the intensity of conflict, the greater negative impact on adolescents' psychological well-being. However, our results show that this is not the case. However, what matters is not the frequency and intensity of the conflicts, but how the conflict is resolved and how they feel about the resolution: when they feel that the solution to the conflict is fair and they feel satisfied with the solution, their psychological well-being level is relatively high; when they unfair or unhappy about the resolution, it is relatively low. This is consistent with the conclusion of previous studies: Leidy, Guerra and Toro (2010) found that moderate conflict can not only enhance individual's ability to cope with events, improve social adaptability, but also help children gain social responsibility and actively explore themselves in the process of transition to adults [19]. Whether parent-child conflict has a posi- 
tive or negative impact on an individual does not lie in the conflict itself, but in parents' and children's coping strategies after the conflict.

This is a great inspiration for parents: it is not terrible to have a conflict with their children, but the resolution of the conflict needs to be very careful. If adolescents often feel that the resolution of the conflict is unfair, and they have negative attitude or extremely dissatisfied with the way to resolve the conflict, it may cause psychological harm to them, and then affect their psychological well-being. Therefore, when there is a conflict between parents and children, we must keep calm, strive to solve the conflict in an appropriate way, and minimize the negative impact of the conflict.

\subsection{Adolescents' Age and Gender in Parent-Child Conflicts}

Our study found that there were differences in the characteristics of conflicts between parents and adolescents of different ages, genders, and their correlation with psychological well-being. Therefore, we should fully consider these differences when dealing with parent-child conflicts. We found that when early adolescents used more personal justification (emphasizing personal choice, hobbies, etc.) to defend for themselves after the conflict, their psychological well-being level was higher; while late adolescents had a higher level of psychological well-being when they used fewer personal justifications. This shows that it is especially important for parents to give early adolescents appropriate autonomy in their personal field and allow them to make decisions in choices of food, clothes, and leisure activities.

There are also gender differences in parent-child conflict. First of all, for boys, using more psychological justifications was related to a low level of psychological well-being; while for girls, there was no such difference. This shows that different strategies should be adopted for boys and girls when guiding them to handle conflicts: we should try not to guide boys to use reasons related to interpersonal relationship and psychological developmental characteristics to defend for themselves. Secondly, for boys, high-intensity conflict was related to poor psychological well-being; while it had no significant impact on girls. Therefore, parents should avoid the escalation of intensity when conflict with male adolescents, so as not to have a serious impact on their psychological well-being.

\section{Conclusions and Limitation}

To sum up: 1) when adolescents felt more fair and happy to the resolution to the conflicts, they have a healthier status of psychological well-being; 2) frequency and intensity of conflicts in general had no significant correlation with adolescents' psychological well-being. However, when male adolescents had a more intensive conflict with their parents, they had a lower level of psychological well-being; 3) adolescents with a higher level of psychological well-being negotiated more on personal issues with their parents; 4) adolescents at different age and gender groups use different justifications when they have conflicts with their parents. 
Thought the current research has reached some interesting and meaningful conclusions, it also has some limitations. Firstly, the sample in the study is relatively small and came from the same big city in China, so it should be cautious when generalizing conclusion in this study. Future study should include bigger and more diverse samples to have a more comprehensive understanding on parent-child relationship. Secondly, parents' education and family income may have impact on parent-child interaction, so future study can involve these variables to have a deeper understanding on this issue.

\section{Conflicts of Interest}

The author declares no conflicts of interest regarding the publication of this paper.

\section{References}

[1] Shek and Daniel, T.L. (1998) A Longitudinal Study of the Relations between Parent-Adolescent Conflict and Adolescent Psychological Well-Being. The Journal of Genetic Psychology, 159, 53-67. https://doi.org/10.1080/00221329809596134

[2] Ge, X., Conger, R.D., Lorenz, F.O. and Simons, R.L. (1994) Parents' Stressful Life Events and Adolescent Depressed Mood. Journal of Health and Social Behavior, 35, 28. https://doi.org/10.2307/2137333

[3] Shek, D.T.L. (1999) Individual and Dyadic Predictors of Family Functioning in a Chinese Context. American Journal of Family Therapy, 27, 49-61. https://doi.org/10.1080/019261899262096

[4] Grotevant, H.D. and Cooper, C.R. (1986) Individuation in Family Relationships: A Perspective on Individual Differences in the Development of Identity and Role-Taking Skill in Adolescence. Human Development, 29, 82-100. https://doi.org/10.1159/000273025

[5] Fang, X.Y., Zhang, J.T., Sun, L. and Liu, Z. (2003) Parent-Adolescent Conflict and Adolescents' Social Adjustment. Chinese Journal of Applied Psychology, 9, 14-21.

[6] Yang, F. and Wu, X.D. (2014) The Relationship among Parenting Style, Parent-Adolescent Conflict and Depression/Anxiety of Junior High School Girls. China Journal of Health and Psychology, 22, 1418-1420.

[7] Yin, H.X. and Shi, G.W. (2010) A Survey of the Research on Parent-Child Conflict of Adolescents. Mental Health Education in Primary and Secondary School, 10, $10-12$.

[8] Nucci, L., Smetana, J.G., Araki, N. and Nakaue, M. (2014) Japanese Adolescents' Disclosure and Information Management with Parents. Child Development, 85, 901-907. https://doi.org/10.1111/cdev.12174

[9] Chen-Gaddini, M. (2012) Chinese Mothers and Adolescents' Views of Authority and Autonomy: A Study of Parent-Adolescent Conflicts in Urban and Rural China. Child Development, 83, 1846-1852. https://doi.org/10.1111/j.1467-8624.2012.01823.x

[10] Helwig, C., Yang, S.G., Nucci, L., Yun, S. and To, S. (2009) Parental Control of the Personal Domain and Adolescent Symptoms of Psychopathology in Urban and Rural China. The Biennial Meeting of the Society for Research in Child Development, Denver, 2-4 April 2009. 
[11] Yau, J. and Smetana, J.G. (1996) Adolescent-Parent Conflict among Chinese Adolescents in Hong Kong. Child Development, 67, 1262-1275. https://doi.org/10.2307/1131891

[12] Yau, J. and Smetana, J.G. (2003) Adolescent-Parent Conflict in Hong Kong and Shenzhen: A Comparison of Youth in Two Cultural Contexts. International Journal of Behavioral Development, 27, 201-211. https://doi.org/10.1080/01650250244000209

[13] Derogatis, L.R. (1994) BSI: Brief Symptom Inventory: Administration, Scoring, and Procedures Manual. National Computer Systems, Minneapolis.

[14] Derogatis, L.R. and Spencer, P.M. (1982) The Brief Symptom Inventory: Administration, Scoring and Procedures Manual I. Clinical Biomedical Research, Baltimore. https://doi.org/10.1037/t00789-000

[15] Steinberg, L. and Silverberg, S.B. (1986) The Vicissitudes of Autonomy in Early Adolescence. Child Development, 57, 841-851. https://doi.org/10.2307/1130361

[16] Wang, M.P. (2006) Adolescents' Beliefs about Parental Authority and Its Relationship with Parenting Style. Journal of Shandong Normal University (Humanities and Social Science), 51, 138-141.

[17] Smetana, J.G. (1989) Adolescents' and Parents' Reasoning about Actual Family Conflict. Child Development, 60, 1052-1067. https://doi.org/10.1111/j.1467-8624.1989.tb03536.x

[18] Lin, C.D. (2002) Developmental Psychology. Hangzhou Education Press, Hangzhou, 397.

[19] Leidy, M.S., Guerra, N.G. and Toro, R.I. (2010) A Review of Family-Based Programs to Prevent Youth Violence among Latinos. Hispanic Journal of Behavioral Sciences, 32, 5-36. https://doi.org/10.1177/0739986309353317 